\title{
MIXED DELAY-DEPENDENT STABILITY OF HIGH-ORDER NEURAL NETWORKS BASED ON A WEAK COUPLING LMI SET
}

\author{
MINGHAO LI ${ }^{1}$, WUNENG ZHOU ${ }^{\otimes 1}$, ZIWEI NI $^{2}$ and MINGJUN WANG ${ }^{1}$
}

(Received 30 October, 2008; revised 11 May, 2009)

\begin{abstract}
This paper deals with the problem of discrete and distributed time-delay exponential stability for deterministic and uncertain stochastic high-order neural networks. The concept of a parameter weak coupling linear matrix inequality set (PWCLMIS) is developed. New results are derived in terms of PWCLMIS. Large mixed time delays can be obtained by using this approach. Furthermore, these results are more general than some previous existence results. Two numerical examples are given to show the merit of the approach.
\end{abstract}

2000 Mathematics subject classification: primary 82C32; secondary 60G52.

Keywords and phrases: neural networks, mixed delays, exponential stability, parameters weak coupling linear matrix inequality set (PWCLMIS).

\section{Introduction}

Because high-order neural networks perform better than traditional first-order neural networks [8], high-order neural networks have been adopted in some fields, for example, associative memories [16], optimization [13] and pattern recognition [2]. To achieve good performances, sufficiency conditions for the stability of a neural network have been studied intensively, see for example [24, 25] and the references therein.

Time delays are frequently the source of instability [1, 5, 6], and sufficient conditions for the stability of high-order neural networks with time delays have been presented in the literature. Both delay-dependent and delay-independent sufficiency conditions have been developed to guarantee the asymptotic, exponential, or absolute stability for high-order neural networks with discrete time delays, see for example [7, $17,26]$.

\footnotetext{
${ }^{1}$ College of Information Science and Technology, Donghua University, Shanghai, PR China; e-mail:wnzhou@dhu.edu.cn.

${ }^{2}$ Department of Computer Science, Xiamen University, Xiamen, PR China; e-mail: zwni@xmu.edu.cn.

(C) Australian Mathematical Society 2010, Serial-fee code 1446-1811/2010\$16.00
} 
Since synaptic transmission is a noisy process brought on by random fluctuations from the release of neurotransmitters and other probabilistic causes [20], investigating neural networks with stochastic perturbations is important both in theory and in practice. Consideration of stochastic inputs that will stabilize or destabilize a neural network [3] has led to some new results being proposed on stability analysis for stochastic neural networks, see for example [12, 19], where discrete time delays have appeared.

In addition to discrete time delays, there is a distribution of propagation delays over a period of time in neural networks. Mixed time delays, which comprise discrete and distributed delays, need to be taken into account when modelling a realistic neural network [18, 27, 28]. The problem of global asymptotic stability for neural networks with mixed time delays has been analysed in [22, 23]. Global asymptotic stability for deterministic stochastic high-order neural networks with mixed time invariant delays has been studied in [20].

For various reasons uncertainties always exist in neural networks, and investigating the robust stability for neural networks with parameter uncertainties is important $[1,5$, 6]. Recently, the robust exponential stability problem for uncertain stochastic neural networks with mixed time delays has been studied in [21], where a linear matrix inequality (LMI) approach has been established.

To the best of the authors' knowledge, large mixed time-varying delay-dependent exponential stability problems for deterministic and uncertain stochastic high-order neural networks have not yet been investigated. In this paper, we develop a novel approach to establish sufficient conditions for high-order neural networks with mixed delays to be globally exponentially stable. This approach is called a parameter weak coupling linear matrix inequality set (PWCLMIS) approach. Assuming a LMI set is coupled by two LMIs, one without system parameters and the other without stability performance parameters (for example, time delays), the system parameters and stability performance parameters are coupled weakly. We call such a LMI set a PWCLMIS. Introducing free-weighting matrices into the PWCLMIS and making some algebraic transformations, we obtain excellent stability performances. Two numerical examples are given to illustrate this characteristic. Furthermore, we encounter both discrete and distributed time-varying delay dependence in this article. Corresponding conditions in [20] are for distributed time-invariant delay dependence only. In addition, we remove some restraints to cover some results in recently published works, such as [7, 17, 20, 26].

1.1. Notation The notation is standard. Throughout this paper, $\mathbb{R}^{n}$ and $\mathbb{R}^{n \times m}$ denote, respectively, the $n$-dimensional Euclidean space and the set of all $n \times m$ real matrices. The superscript " $T$ " denotes matrix transposition and the notation $X \geq Y$ (respectively, $X>Y$ ), where $X$ and $Y$ are symmetric matrices, means that $X-Y$ is positive semidefinite (respectively, positive definite). We use $I_{n}$ to denote the $n \times n$ identity matrix and use $|\cdot|$ to denote the Euclidean norm in $\mathbb{R}^{n}$. If $A$ is a matrix, denote by $\|A\|$ its operator norm, that is, $\|A\|=\sup \{|A x|:|x|=1\}=\sqrt{\lambda_{\max }\left(A^{\mathrm{T}} A\right)}$ where $\lambda_{\max }(\cdot)$ 
(respectively, $\left.\lambda_{\min }(\cdot)\right)$ means the largest (respectively, smallest) eigenvalue of $A$. Here $l_{2}[0, \infty)$ is the space of square integrable vectors. Moreover, let $\left(\Omega, \mathcal{F},\left\{\mathcal{F}_{t}\right\}_{t \geq 0}, P\right)$ be a complete probability space with a filtration $\left\{\mathcal{F}_{t}\right\}_{t \geq 0}$ satisfying the usual conditions (that is, the filtration contains all $P$-null sets and is right continuous). Denote by $L_{\mathcal{F}_{0}}^{P}\left([-h, 0] ; \mathbb{R}^{n}\right)$ the family of all $\mathcal{F}_{0}$-measurable $C\left([-h, 0] ; \mathbb{R}^{n}\right)$-valued random variables $\xi=\{\xi(\theta):-h \leq \theta \leq 0\}$ such that $\sup _{-h \leq \theta \leq 0} \mathbb{E}|\xi(\theta)|^{p}<\infty$ where $\mathbb{E}\{\cdot\}$ stands for the mathematical expectation operator with respect to the given probability measure $P$. The shorthand $\operatorname{diag}\left\{M_{1}, M_{2}, \ldots, M_{N}\right\}$ denotes a block-diagonal matrix with diagonal blocks being the matrices $M_{1}, M_{2}, \ldots, M_{N}$. Sometimes, the arguments of a function or a matrix will be omitted in the analysis when no confusion can arise.

\section{Preliminaries and problem formulation}

We consider high-order neural networks with mixed time delays

$$
\begin{aligned}
d x(t)= & {\left[-A x(t)+W_{0} f(x(t))+W_{1} f(x(t-h(t)))+W_{2} \int_{t-\tau(t)}^{t} f(x(s)) d s\right] d t } \\
& +\sigma(t, x(t), x(t-h(t))) d w(t),
\end{aligned}
$$

where

$$
\begin{gathered}
x(t)=\left(x_{1}(t), x_{2}(t), \ldots, x_{n}(t)\right)^{\mathrm{T}} \in \mathbb{R}^{n}, \\
f(x(t))=\left(f_{1}(x(t)), f_{2}(x(t)), \ldots, f_{L}(x(t))\right)^{\mathrm{T}} \in \mathbb{R}^{L}, \\
f(x(t-h(t)))=\left(f_{1}(x(t-h(t))), f_{2}(x(t-h(t))), \ldots, f_{L}(x(t-h(t)))\right)^{\mathrm{T}} \in \mathbb{R}^{L}, \\
A=\operatorname{diag}\left\{a_{1}, a_{2}, \ldots, a_{n}\right\}>0, \quad W_{0}=\left[w_{0 i j}\right]_{n \times L} \in \mathbb{R}^{n \times L}, \\
W_{1}=\left[w_{1 i j}\right]_{n \times L} \in \mathbb{R}^{n \times L}, \quad f_{j}(x(t))=\prod_{k \in I_{j}}\left[g_{k}\left(x_{k}(t)\right)\right]^{d_{k}(j)} .
\end{gathered}
$$

Here $I_{i}(i=1,2, \ldots, L)$ is a subset of $\{1,2, \ldots, n\}, d_{k}(j)$ a positive integer, $g_{i}(\cdot)$ the activation function with $g_{i}(0)=0, x(t)$ the state vector associated with the $n$ neurons, and the matrix $A=\operatorname{diag}\left\{a_{1}, a_{2}, \ldots, a_{n}\right\}$ has positive entries $a_{i}>0$. The matrices $W_{0}, W_{1}$ and $W_{2}$ are, respectively, the connection weight matrix, the discretely delayed connection weight matrix, and the distributively delayed connection weight matrix. Here $f(x(\cdot))$ is a product of $L$ activation functions that reflects high-order characteristics. The scalars $h(t)>0$ and $\tau(t)>0$ are the unknown discrete time delay and unknown distributed time delay, respectively. The scalars $h(t)$ and $\tau(t)$ satisfy

$$
\begin{cases}0 \leq \tau(t) \leq \tau, & \dot{\tau}(t) \leq d_{\tau}, \\ 0 \leq h(t) \leq h, & \dot{h}(t) \leq d_{h}, \\ \tau_{0}=\max (\tau, h) . & \end{cases}
$$

REMARK 1 . The $d_{\tau}<1$ and $d_{h}<1$ constraints that usually appear in the literature have been removed. 
REMARK 2. There are two differences from [20] in our formulation. First, the discrete time delay $h$ is a time-varying delay here but a time-invariant delay in [20]. Second, the scalar $\tau(t)=\tau>0$ is the unknown distributed time-varying delay in this note but a known constant distributed time delay in [20].

The stochastic disturbance $w(t)=\left[w_{1}(t), w_{2}(t), \ldots, w_{m}(t)\right]^{\mathrm{T}} \in \mathbb{R}^{m}$ is a Brownian motion defined on the complete probability space $\left(\Omega, \mathcal{F},\left\{\mathcal{F}_{t}\right\}_{t \geq 0}, P\right)$. Assume that $\sigma: \mathbb{R}^{+} \times \mathbb{R}^{n} \times \mathbb{R}^{n} \rightarrow \mathbb{R}^{n}$ is locally Lipschitz continuous and satisfies a linear growth condition [11]. Moreover, $\sigma$ satisfies

$$
\begin{aligned}
\operatorname{trace}[ & \left.\sigma^{\mathrm{T}}(t, x(t), x(t-h(t))) \sigma(t, x(t), x(t-h(t)))\right] \\
& \leq\left|\Sigma_{1} x(t)\right|^{2}+\left|\Sigma_{2} x(t-h(t))\right|^{2},
\end{aligned}
$$

where $\Sigma_{1}$ and $\Sigma_{2}$ are known constant matrices of appropriate dimension.

REMARK 3 ([20]). The condition (2.3) imposed on the stochastic disturbance term, $\sigma^{\mathrm{T}}(t, x(t), x(t-h(t)))$, has been used in recent papers dealing with stochastic neural networks, see [12] and references therein.

Parameter uncertainties and stochastic perturbations are common sources of disturbances in neural networks. We model the uncertain stochastic neural networks with mixed time delays as

$$
\begin{aligned}
d x(t)= & {\left[-(A+\Delta A) x(t)+\left(W_{0}+\Delta W_{0}\right) f(x(t))+\left(W_{1}+\Delta W_{1}\right) f(x(t-h(t)))\right.} \\
& \left.+W_{2} \int_{t-\tau(t)}^{t} f(x(s)) d s\right] d t+\sigma(t, x(t), x(t-h(t))) d w(t)
\end{aligned}
$$

where $\Delta A, \Delta W_{0}$ and $\Delta W_{1}$ are unknown matrices representing time-varying parameter uncertainties and satisfying the admissible condition

$$
\left[\begin{array}{lll}
\Delta A & \Delta W_{0} & \Delta W_{1}
\end{array}\right]=M F\left[\begin{array}{lll}
N_{1} & N_{2} & N_{3}
\end{array}\right],
$$

where $M, N_{1}, N_{2}$ and $N_{3}$ are known real constant matrices, and $F$ is the unknown time-varying matrix-valued function subject to

$$
F^{\mathrm{T}} F \leq I \text {. }
$$

We make the following assumptions throughout this paper [17].

Asumption 2.1. There exist constants $\mu_{i}>0$ such that

$$
\left|g_{i}(x)\right| \leq \mu_{i}|x|, \quad \forall x \in \mathbb{R}, i=1,2, \ldots, n .
$$

ASUMPTION 2.2. For all $g_{i}(\cdot)$

$$
\left|g_{i}(x)\right| \leq 1, \quad \forall x \in \mathbb{R}, i=1,2, \ldots, n .
$$


Denote by $x(t ; \xi)$ the state trajectory of the neural network (2.1) or (2.4) from the initial data $x(\theta)=\xi(\theta)$ on $-\tau_{0} \leq \theta \leq 0$ in $L_{\mathcal{F}_{0}}^{2}\left(\left[-\tau_{0}, 0\right] ; \mathbb{R}^{n}\right)$. According to [11], the system (2.1) or (2.4) admits a trivial solution $x(t ; 0) \equiv 0$ corresponding to the initial data $\xi=0$.

Before proceeding further, we introduce the definition of global exponential stability for the uncertain stochastic neural network (2.1) or (2.4) with discrete and distributed time delays as follows.

DEFINITION 2.3. For the neural network (2.1) or (2.4) and every

$$
\xi \in L_{\mathcal{F}_{0}}^{2}\left([-h, 0] ; \mathbb{R}^{n}\right)
$$

the trivial solution (equilibrium point) is robustly, globally, exponentially stable in mean square if there exist positive constants $\beta>0$ and $\mu>0$ such that every solution $x(t ; \xi)$ of (2.1) or (2.4) satisfies

$$
\mathbb{E}\left\{\|x(t ; \xi)\|^{2}\right\} \leq \mu e^{-\beta t} \sup _{-\tau_{0} \leq s \leq 0} \mathbb{E}\left\{\|\xi(s)\|^{2}\right\}, \quad \forall t>0 .
$$

The main objective of this paper is to establish LMI-based stability criteria to guarantee the high-order uncertain stochastic neural network is robustly exponentially stable with mixed time delays and the admissible time delays of stability condition are large.

\section{Main results}

Before deriving the main results, we give the following lemmas.

Lemma 3.1. Let $x \in \mathbb{R}^{n}, y \in \mathbb{R}^{n}$ and $\varepsilon>0$. Then $x^{\mathrm{T}} y+y^{\mathrm{T}} x \leq \varepsilon x^{\mathrm{T}} x+\varepsilon^{-1} y^{\mathrm{T}} y$.

LEMMA 3.2 (Boyd et al. [4]). Given constant matrices $\Omega_{1}, \Omega_{2}$ and $\Omega_{3}$ where $\Omega_{1}=\Omega_{1}^{\mathrm{T}}$ and $0<\Omega_{2}=\Omega_{2}^{\mathrm{T}}$, we then have $\Omega_{1}+\Omega_{3}^{\mathrm{T}} \Omega_{2}^{-1} \Omega_{3}<0$ if and only if

$$
\left[\begin{array}{rr}
\Omega_{1} & \Omega_{3}^{\mathrm{T}} \\
\Omega_{3} & -\Omega_{2}
\end{array}\right]<0 \quad \text { or } \quad\left[\begin{array}{rr}
-\Omega_{2} & \Omega_{3} \\
\Omega_{3}^{\mathrm{T}} & \Omega_{1}
\end{array}\right]<0 .
$$

LEMMA 3.3 (Gu [10]). For any positive-definite matrix $M$, scalar $\gamma>0$, vector function $w:[0, \gamma] \rightarrow \mathbb{R}^{n}$ such that the integrations concerned are well defined, we have

$$
\left(\int_{0}^{\gamma} w(s) d s\right)^{\mathrm{T}} M\left(\int_{0}^{\gamma} w(s) d s\right) \leq \gamma\left(\int_{0}^{\gamma} w^{\mathrm{T}}(s) M w(s) d s\right) .
$$

LEMMA 3.4 (Ren and Cao [17]). Let $f(x)=\left(f_{1}(x), f_{2}(x), \ldots, f_{L}(x)\right)^{\mathrm{T}} \in \mathbb{R}^{L}$ where $L$ is an integer, and $\Sigma_{\mu}=\operatorname{diag}\left\{\mu_{1}, \ldots, \mu_{n}\right\}$ where $\mu_{i}$ is defined in Assumption 2.1. Then, from Assumptions 2.1 and 2.2,

$$
f^{\mathrm{T}}(x) f(x) \leq L x^{\mathrm{T}} \Sigma_{\mu} \Sigma_{\mu} x .
$$


LEMMA 3.5 (Petersen [15]). Given matrices $\Omega, \Gamma$ and $\Xi$ with appropriate dimensions and with $\Omega$ symmetrical, then

$$
\Omega+\Gamma F \Xi+\Xi^{\mathrm{T}} F^{\mathrm{T}} \Gamma^{\mathrm{T}}<0
$$

for any $F$ satisfying $F^{\mathrm{T}} F \leq I$, if and only if there exists a scalar $\varepsilon>0$ such that

$$
\Omega+\varepsilon \Gamma \Gamma^{\mathrm{T}}+\varepsilon^{-1} \Xi^{\mathrm{T}} \Xi<0 .
$$

3.1. Exponential stability for deterministic systems The following theorem provides a sufficient condition for robust global exponential stability in mean square for the network dynamics system (2.1).

THEOREM 3.6. Consider the dynamics of the high-order stochastic delayed neural network (2.1). The system is robustly, globally, exponentially stable in mean square if there exist scalars $\rho>0, \varepsilon_{i}>0(i=1,2,3)$ and matrices $P>0, Q_{1}>0, Q_{2}>0$, $Z_{j}>0(j=1,2,3,4)$,

$$
X^{\mathrm{T}}=\left[X_{1}^{\mathrm{T}}, X_{2}^{\mathrm{T}}, X_{3}^{\mathrm{T}}, X_{4}^{\mathrm{T}}, X_{5}^{\mathrm{T}}\right] \quad \forall X=H, J, K, L, R, S
$$

such that the PWCLMIS

$$
\Psi_{1}=\left[\begin{array}{cccccccc}
\Omega_{11} & P W_{0} & \Omega_{13} & P W_{1} & P W_{2} & \rho \Sigma_{1}^{\mathrm{T}} & 0 & 0 \\
* & -\varepsilon_{1} I & 0 & 0 & 0 & 0 & 0 & 0 \\
* & * & -\varepsilon_{1} I & 0 & 0 & 0 & 0 & 0 \\
* & * & * & -\varepsilon_{2} I & 0 & 0 & 0 & 0 \\
* & * & * & * & -\varepsilon_{3} I & 0 & 0 & 0 \\
* & * & * & * & * & -\rho I & 0 & 0 \\
* & * & * & * & * & * & \Omega_{77} & \rho \Sigma_{2}^{\mathrm{T}} \\
* & * & * & * & * & * & * & -\rho I
\end{array}\right]<0,
$$

holds, where

$$
\begin{aligned}
& \Omega_{11}=-A P-P A+Q_{1}+Q_{2}, \quad \Omega_{13}=\varepsilon_{1} L^{1 / 2} \Sigma_{\mu}, \\
& \Omega_{77}=\varepsilon_{2} L \Sigma_{\mu} \Sigma_{\mu}-\left(1-d_{h}\right) Q_{2}, \\
& \Phi=\Phi_{1}+\Phi_{2}+\Phi_{2}^{\mathrm{T}}, \quad \Phi_{1}=\operatorname{diag}\left\{0,-\left(1-d_{\tau}\right) Q_{1}, 0,0,0\right\}, \\
& \Phi_{2}=\left[\begin{array}{lllll}
H+K+L+S & -H+J & -J-K & -L+R & -R-S
\end{array}\right] \text {. }
\end{aligned}
$$


PROOF. Define a Lyapunov-Krasovskii functional $V(t, x(t))$ by

$$
\begin{aligned}
V(t, x(t))= & x^{\mathrm{T}}(t) P x(t)+\int_{t-\tau(t)}^{t} x^{\mathrm{T}}(s) Q_{1} x(s) d s+\int_{t-h(t)}^{t} x^{\mathrm{T}}(s) Q_{2} x(s) d s \\
& +\int_{-\tau}^{0} \int_{t+\theta}^{0} x^{\mathrm{T}}(s)\left(\varepsilon_{3} \tau L \Sigma_{\mu} \Sigma_{\mu}\right) x(s) d s d \theta \\
& +\int_{-\tau}^{0} \int_{t+\theta}^{0} \dot{x}^{\mathrm{T}}(s)\left(Z_{1}+Z_{2}\right) \dot{x}(s) d s d \theta \\
& +\int_{-h}^{0} \int_{t+\theta}^{0} \dot{x}^{\mathrm{T}}(s)\left(Z_{3}+Z_{4}\right) \dot{x}(s) d s d \theta .
\end{aligned}
$$

By Itô's differential formula [9], the stochastic derivative of $V(t, x(t))$ along (2.1) is

$$
\begin{aligned}
d V(t, x(t)) & \\
= & {\left[x^{\mathrm{T}}(t) P \sigma(t, x(t), x(t-h(t)))+\sigma^{\mathrm{T}}(t, x(t), x(t-h(t))) P x(t)\right] d w(t) } \\
& +\mathcal{L} V(t, x(t)) d t,
\end{aligned}
$$

where

$$
\begin{aligned}
\mathcal{L} V(t, x(t))= & x^{\mathrm{T}}(t)\left(-A^{\mathrm{T}} P-P A+Q_{1}+Q_{2}\right) x(t) \\
& -(1-\dot{\tau}(t)) x^{\mathrm{T}}(t-\tau(t)) Q_{1} x(t-\tau(t)) \\
& -(1-\dot{h}(t)) x^{\mathrm{T}}(t-h(t)) Q_{2} x(t-h(t))+2 x^{\mathrm{T}}(t) P W_{0} f(x(t)) \\
& +2 x^{\mathrm{T}}(t) P W_{1} f(x(t-h(t)))+2 x^{\mathrm{T}}(t) P W_{2} \int_{t-\tau}^{t} f(x(s)) d s \\
& -\int_{t-\tau}^{t} x^{\mathrm{T}}(s)\left(\varepsilon_{3} \tau L \Sigma_{\mu} \Sigma_{\mu}\right) x(s) d s-\int_{t-\tau}^{t} \dot{x}^{\mathrm{T}}(s)\left(Z_{1}+Z_{2}\right) \dot{x}(s) d s \\
& -\int_{t-h}^{t} \dot{x}^{\mathrm{T}}(s)\left(Z_{3}+Z_{4}\right) \dot{x}(s) d s \\
& +\operatorname{trace}\left[\sigma^{\mathrm{T}}(t, x(t), x(t-h(t))) P \sigma(t, x(t), x(t-h(t)))\right] .
\end{aligned}
$$

According to conditions (2.3) and (3.2),

$$
\begin{aligned}
\operatorname{trace} & {\left[\sigma^{\mathrm{T}}(t, x(t), x(t-h(t))) P \sigma(t, x(t), x(t-h(t)))\right] } \\
& \leq \rho\left[x^{\mathrm{T}}(t) \Sigma_{1}^{\mathrm{T}} \Sigma_{1} x(t)+x^{\mathrm{T}}(t-h(t)) \Sigma_{2}^{\mathrm{T}} \Sigma_{2} x(t-h(t))\right] .
\end{aligned}
$$


By Lemmas 3.1 and 3.4,

$$
\begin{aligned}
2 x^{\mathrm{T}}(t) P W_{0} f(x(t)) \leq & \varepsilon_{1} f^{\mathrm{T}}(x(t)) f(x(t))+\varepsilon_{1}^{-1} x^{\mathrm{T}}(t) P W_{0} W_{0}^{\mathrm{T}} P x(t) \\
\leq & x^{\mathrm{T}}(t)\left(\varepsilon_{1} L \Sigma_{\mu} \Sigma_{\mu}+\varepsilon_{1}^{-1} P W_{0} W_{0}^{\mathrm{T}} P\right) x(t), \\
2 x^{\mathrm{T}}(t) P W_{1} f(x(t-h(t))) \leq & \varepsilon_{2} f^{\mathrm{T}}(x(t-h(t))) f(x(t-h(t))) \\
& +\varepsilon_{2}^{-1} x^{\mathrm{T}}(t) P W_{1} W_{1}^{\mathrm{T}} P x(t) \\
\leq & \varepsilon_{2} x^{\mathrm{T}}(t-h(t)) L \Sigma_{\mu} \Sigma_{\mu} x(t-h(t)) \\
& +\varepsilon_{2}^{-1} x^{\mathrm{T}}(t) P W_{1} W_{1}^{\mathrm{T}} P x(t)
\end{aligned}
$$

and

$$
\begin{aligned}
2 x^{\mathrm{T}}(t) P W_{2} \int_{t-\tau}^{t} f(x(s)) d s \leq & \varepsilon_{3}\left(\int_{t-\tau}^{t} f(x(s)) d s\right)^{\mathrm{T}} \int_{t-\tau}^{t} f(x(s)) d s \\
& +\varepsilon_{3}^{-1} x^{\mathrm{T}}(t) P W_{2} W_{2}^{\mathrm{T}} P x(t),
\end{aligned}
$$

where $\varepsilon_{1}, \varepsilon_{2}, \varepsilon_{3}>0$.

By Lemmas 3.3 and 3.4, we have that

$$
\begin{aligned}
& \varepsilon_{3}\left(\int_{t-\tau}^{t} f(x(s)) d s\right)^{\mathrm{T}} \int_{t-\tau}^{t} f(x(s)) d s \leq \varepsilon_{3} \tau \int_{t-\tau}^{t} f^{\mathrm{T}}(x(s)) f(x(s)) d s \\
& \leq \int_{t-\tau}^{t} x^{\mathrm{T}}(s)\left(\varepsilon_{3} \tau L \Sigma_{\mu} \Sigma_{\mu}\right) x(s) d s .
\end{aligned}
$$

Substituting (3.8)-(3.12) into (3.7) and taking note of (2.2) gives

$$
\mathcal{L} V(t, x(t)) \leq \mathcal{L} V_{1}(t, x(t))+\mathcal{L} V_{2}(t, x(t)),
$$

where

$$
\mathcal{L} V_{1}(t, x(t))=\xi_{1}^{\mathrm{T}}(t) \Xi \xi_{1}(t),
$$

for

$$
\begin{gathered}
\xi_{1}(t)=\left[\begin{array}{ll}
x^{\mathrm{T}}(t) \quad x^{\mathrm{T}}(t-h(t))
\end{array}\right]^{\mathrm{T}}, \quad \Xi=\operatorname{diag}\left\{\Xi_{11}, \Xi_{22}\right\}, \\
\Xi_{11}=-A P-P A+Q_{1}+Q_{2}+\rho \Sigma_{1}^{\mathrm{T}} \Sigma_{1}+\varepsilon_{1} L \Sigma_{\mu} \Sigma_{\mu}+\varepsilon_{1}^{-1} P W_{0} W_{0}^{\mathrm{T}} P \\
+\varepsilon_{2}^{-1} P W_{1} W_{1}^{\mathrm{T}} P+\varepsilon_{3}^{-1} P W_{2} W_{2}^{\mathrm{T}} P, \\
\Xi_{22}=\varepsilon_{2} L \Sigma_{\mu} \Sigma_{\mu}+\rho \Sigma_{2}^{\mathrm{T}} \Sigma_{2}-\left(1-d_{h}\right) Q_{2}
\end{gathered}
$$

and

$$
\begin{aligned}
\mathcal{L} V_{2}(t, x(t))= & -\left(1-d_{\tau}\right) x^{\mathrm{T}}(t-\tau(t)) Q_{1} x(t-\tau(t))-\int_{t-\tau}^{t} \dot{x}^{\mathrm{T}}(s)\left(Z_{1}+Z_{2}\right) \dot{x}(s) d s \\
& -\int_{t-h}^{t} \dot{x}^{\mathrm{T}}(s)\left(Z_{3}+Z_{4}\right) \dot{x}(s) d s .
\end{aligned}
$$


It follows from the Schur complement lemma (Lemma 3.2) that (3.3) implies that $\Xi<0$, so we obtain

$$
\mathcal{L} V_{1}(t, x(t))<0
$$

Next, we observe $\mathcal{L} V_{2}(t, x(t))$.

By the Leibniz-Newton formula, for any matrices $H, J, K, L, R$ and $S$ with appropriate dimensions:

$$
\begin{gathered}
2 \xi_{2}^{\mathrm{T}}(t) H\left[x(t)-x(t-\tau(t))-\int_{t-\tau(t)}^{t} \dot{x}(s) d s\right]=0, \\
2 \xi_{2}^{\mathrm{T}}(t) J\left[x(t-\tau(t))-x(t-\tau)-\int_{t-\tau}^{t-\tau(t)} \dot{x}(s) d s\right]=0, \\
2 \xi_{2}^{\mathrm{T}}(t) K\left[x(t)-x(t-\tau)-\int_{t-\tau}^{t} \dot{x}(s) d s\right]=0, \\
2 \xi_{2}^{\mathrm{T}}(t) L\left[x(t)-x(t-h(t))-\int_{t-h(t)}^{t} \dot{x}(s) d s\right]=0, \\
2 \xi_{2}^{\mathrm{T}}(t) R\left[x(t-h(t))-x(t-h)-\int_{t-h}^{t-h(t)} \dot{x}(s) d s\right]=0, \\
2 \xi_{2}^{\mathrm{T}}(t) S\left[x(t)-x(t-h)-\int_{t-h}^{t} \dot{x}(s) d s\right]=0,
\end{gathered}
$$

where

$$
\xi_{2}(t)=\left[\begin{array}{lllll}
x^{\mathrm{T}}(t) & x^{\mathrm{T}}(t-\tau(t)) & x^{\mathrm{T}}(t-\tau) & x^{\mathrm{T}}(t-h(t)) & x^{\mathrm{T}}(t-h)
\end{array}\right]^{\mathrm{T}} .
$$

On adding the left-hand sides to (3.15), we obtain

$$
\begin{aligned}
\mathcal{L} V_{2}(t, & x(t)) \\
\leq & -\left(1-d_{\tau}\right) x^{\mathrm{T}}(t-\tau(t)) Q_{1} x(t-\tau(t))+2 \xi_{2}^{\mathrm{T}}(t) H[x(t)-x(t-\tau(t))] \\
& +2 \xi_{2}^{\mathrm{T}}(t) J[x(t-\tau(t))-x(t-\tau)]+2 \xi_{2}^{\mathrm{T}}(t) K[x(t)-x(t-\tau)] \\
& +\tau \xi_{2}^{\mathrm{T}}(t)\left(H Z_{1}^{-1} H^{\mathrm{T}}+J Z_{1}^{-1} J^{\mathrm{T}}+K Z_{2}^{-1} K^{\mathrm{T}}\right) \xi_{2}^{\mathrm{T}}(t) \\
& -\int_{t-\tau(t)}^{t}\left[\dot{x}^{\mathrm{T}}(s) Z_{1}+\xi_{2}^{\mathrm{T}}(t) H\right] Z_{1}^{-1}\left[Z_{1}^{\mathrm{T}} \dot{x}(s)+H^{\mathrm{T}} \xi_{2}(t)\right] d s \\
& -\int_{t-\tau}^{t-\tau(t)}\left[\dot{x}^{\mathrm{T}}(s) Z_{1}+\xi_{2}^{\mathrm{T}}(t) J\right] Z_{1}^{-1}\left[Z_{1}^{\mathrm{T}} \dot{x}(s)+J^{\mathrm{T}} \xi_{2}(t)\right] d s
\end{aligned}
$$




$$
\begin{aligned}
& -\int_{t-\tau}^{t}\left[\dot{x}^{\mathrm{T}}(s) Z_{2}+\xi_{2}^{\mathrm{T}}(t) K\right] Z_{2}^{-1}\left[Z_{2}^{\mathrm{T}} \dot{x}(s)+K^{\mathrm{T}} \xi_{2}(t)\right] d s \\
& +2 \xi_{2}^{\mathrm{T}}(t) L[x(t)-x(t-h(t))]+2 \xi_{2}^{\mathrm{T}}(t) S[x(t)-x(t-h)] \\
& +2 \xi_{2}^{\mathrm{T}}(t) R[x(t-h(t))-x(t-h)] \\
& +h \xi_{2}^{\mathrm{T}}(t)\left(L Z_{3}^{-1} L^{\mathrm{T}}+R Z_{3}^{-1} R^{\mathrm{T}}+S Z_{4}^{-1} S^{\mathrm{T}}\right) \xi_{2}^{\mathrm{T}}(t) \\
& -\int_{t-h(t)}^{t}\left[\dot{x}^{\mathrm{T}}(s) Z_{3}+\xi_{2}^{\mathrm{T}}(t) L\right] Z_{3}^{-1}\left[Z_{3}^{\mathrm{T}} \dot{x}(s)+L^{\mathrm{T}} \xi_{2}(t)\right] d s \\
& -\int_{t-h}^{t-h(t)}\left[\dot{x}^{\mathrm{T}}(s) Z_{3}+\xi_{2}^{\mathrm{T}}(t) R\right] Z_{3}^{-1}\left[Z_{3}^{\mathrm{T}} \dot{x}(s)+R^{\mathrm{T}} \xi_{2}(t)\right] d s \\
& -\quad \int_{t-h}^{t}\left[\dot{x}^{\mathrm{T}}(s) Z_{4}+\xi_{2}^{\mathrm{T}}(t) S\right] Z_{4}^{-1}\left[Z_{4}^{\mathrm{T}} \dot{x}(s)+S^{\mathrm{T}} \xi_{2}(t)\right] d s \\
& \leq \xi_{2}^{\mathrm{T}}(t)\left[\Phi+\tau H Z_{1}^{-1} H^{\mathrm{T}}+\tau J Z_{1}^{-1} J^{\mathrm{T}}+\tau K Z_{2}^{-1} K^{\mathrm{T}}+h L Z_{3}^{-1} L^{\mathrm{T}}\right. \\
& \left.\quad+h R Z_{3}^{-1} R^{\mathrm{T}}+h S Z_{4}^{-1} S^{\mathrm{T}}\right] \xi_{2}(t)
\end{aligned}
$$

From (3.4), by the Schur complement lemma (Lemma 3.2), we obtain

$$
\begin{aligned}
\Phi & +\tau H Z_{1}^{-1} H^{\mathrm{T}}+\tau J Z_{1}^{-1} J^{\mathrm{T}}+\tau K Z_{2}^{-1} K^{\mathrm{T}}+h L Z_{3}^{-1} L^{\mathrm{T}}+h R Z_{3}^{-1} R^{\mathrm{T}} \\
& +h S Z_{4}^{-1} S^{\mathrm{T}}<0
\end{aligned}
$$

so that

$$
\mathcal{L} V_{2}(t, x(t))<0 .
$$

Thus, from (3.13), (3.14), (3.15), (3.17) and (3.18),

$$
\mathcal{L} V(t, x(t))=\mathcal{L} V_{1}(t, x(t))+\mathcal{L} V_{2}(t, x(t))<0 .
$$

If $\lambda_{1}:=\min _{i \in S}\left\{\lambda_{\min }\left(-\Psi_{1}\right)\right\}, \lambda_{2}:=\min _{i \in S}\left\{\lambda_{\min }\left(-\Psi_{2}\right)\right\}$ and

$\mathbb{E}\{\mathcal{L} V(t, x(t))\} \leq-\lambda_{1} \mathbb{E}\left\{\|x(t)\|^{2}\right\}-\lambda_{2} \mathbb{E}\left\{\|x(t)\|^{2}\right\} \leq-\lambda_{1} \mathbb{E}\left\{\|x(t)\|^{2}\right\}<0$.

The function

$$
W(t, x(t)):=e^{k t} V(t, x(t)), \quad k>0,
$$

has infinitesimal operator $\mathcal{L}$ given by

$$
\mathcal{L} W(t, x(t))=k e^{k t} V(t, x(t))+e^{k t} \mathcal{L} V(t, x(t)) .
$$


Let $\phi(t)=\left[\xi_{2}^{\mathrm{T}}(t) \dot{x}^{\mathrm{T}}(s)\right]^{\mathrm{T}}$. By the generalized Itô formula we can obtain from (3.21) that

$$
\begin{aligned}
\mathbb{E}\{W(t, x(t))\}= & \mathbb{E}\{W(0, x(0))\}+\int_{0}^{t} k e^{k t} \mathbb{E}\{V(s, x(s))\} d s \\
& +\int_{0}^{t} e^{k t} \mathbb{E}\{\mathcal{L} V(s, x(s))\} d s \\
\leq & \lambda_{\max }(P) \mathbb{E}\left\{\|x(0)\|^{2}\right\}+k e^{k t}\left[\lambda_{\max }(P)+\tau \lambda_{\max }\left(Q_{1}\right)+h \lambda_{\max }\left(Q_{2}\right)\right. \\
& \left.+\tau \lambda_{\max }\left(Z_{1}+Z_{2}\right)+h \lambda_{\max }\left(Z_{3}+Z_{4}\right)+\varepsilon_{3} \tau^{2} L \Sigma_{\mu} \Sigma_{\mu}\right] \\
& \times \int_{0}^{\tau_{0}} \mathbb{E}\left\{\|\phi(s)\|^{2}\right\} d s-\lambda_{1} e^{k t} \int_{0}^{\tau_{0}} \mathbb{E}\left\{\|x(s)\|^{2}\right\} d s \\
\leq & \eta \sup _{-\tau_{0} \leq s \leq 0} \mathbb{E}\left\{\|\phi(t)\|^{2}\right\},
\end{aligned}
$$

where

$$
\begin{aligned}
\eta= & \lambda_{\max }(P)+\tau_{0} k e^{k t}\left[\lambda_{\max }(P)+\tau \lambda_{\max }\left(Q_{1}\right)+h \lambda_{\max }\left(Q_{2}\right)+\tau \lambda_{\max }\left(Z_{1}+Z_{2}\right)\right. \\
& \left.+h \lambda_{\max }\left(Z_{3}+Z_{4}\right)+\varepsilon_{3} \tau^{2} L \Sigma_{\mu} \Sigma_{\mu}\right] .
\end{aligned}
$$

Also, it is easy to see that

$$
\mathbb{E}\{V(t, x(t))\} \geq \lambda_{\min }(P) \mathbb{E}\left\{\|x(t)\|^{2}\right\} .
$$

From (3.23) and (3.24), if follows that

$$
\mathbb{E}\left\{\|x(t)\|^{2}\right\} \leq \lambda_{\min }^{-1}(P) \eta e^{-k t} \sup _{-\tau_{0} \leq s \leq 0} \mathbb{E}\left\{\|\phi(s)\|^{2}\right\} .
$$

This completes the proof.

REMARK 4. Theorem 3.6 gives a new stability criteria for system (2.1). We define a new Lyapunov-Krasovskii functional (3.5) which makes full use of the information about discrete and distributed time delays to derive the result. Furthermore, some novel techniques have been exploited in the calculation of the time derivative of $V(t)$. First, no assumptions about $Q_{1}$ and $Q_{2}$ are involved in the system (2.1). However, $Q_{1}=\varepsilon_{2} L \Sigma_{\mu} \Sigma_{\mu}+\rho \Sigma_{2}^{\mathrm{T}} \Sigma_{2}$ and $Q_{2}=\varepsilon_{3} \tau L \Sigma_{\mu} \Sigma_{\mu}$ have been adopted in [20]. Thus, the presented criteria have the potential to yield more general results. Second, our result concerns exponential stability while the result in [20] relates to asymptotic stability. So our result gives faster convergence. Finally, the PWCLMIS presented in [14] has been employed in this note.

If only discrete time delay appears in the neural network, (2.1) can be simplified to

$$
\begin{aligned}
d x(t)= & {\left[-A x(t)+W_{0} f(x(t))+W_{1} f(x(t-h(t)))\right] d t } \\
& +\sigma(t, x(t), x(t-h(t))) d w(t) .
\end{aligned}
$$


The stability issue for stochastic high-order neural networks with discrete delays has been investigated in [20], and the following corollary provides a more universal result.

COROllary 3.7. Consider the dynamics of the neural network (3.26). The system is robustly, globally, exponentially stable in mean square if there exist scalars $\rho>0$, $\varepsilon_{1}>0$ and $\varepsilon_{2}>0$ and matrices $P>0, Q_{2}>0, Z_{3}>0$ and $Z_{4}>0$,

$$
L=\left[\begin{array}{l}
L_{1} \\
L_{2} \\
L_{3}
\end{array}\right], \quad R=\left[\begin{array}{l}
R_{1} \\
R_{2} \\
R_{3}
\end{array}\right], \quad S=\left[\begin{array}{l}
S_{1} \\
S_{2} \\
S_{3}
\end{array}\right]
$$

such that the following PWCLMIS holds:

$$
\begin{gathered}
{\left[\begin{array}{ccccccc}
\bar{\Omega}_{11} & P W_{0} & \Omega_{13} & P W_{1} & \rho \Sigma_{1}^{\mathrm{T}} & 0 & 0 \\
* & -\varepsilon_{1} I & 0 & 0 & 0 & 0 & 0 \\
* & * & -\varepsilon_{1} I & 0 & 0 & 0 & 0 \\
* & * & * & -\varepsilon_{2} I & 0 & 0 & 0 \\
* & * & * & * & -\rho I & 0 & 0 \\
* & * & * & * & * & \Omega_{77} & \rho \Sigma_{2}^{\mathrm{T}} \\
* & * & * & * & * & * & -\rho I
\end{array}\right]<0,} \\
\\
{\left[\begin{array}{ccccc}
\bar{\Phi} & h L & h R & h S \\
* & -h Z_{3} & 0 & 0 \\
* & * & -h Z_{3} & 0 \\
* & * & * & -h Z_{4}
\end{array}\right]<0,}
\end{gathered}
$$

where

$$
\bar{\Omega}_{11}=-A P-P A+Q_{2}, \quad \bar{\Phi}=\bar{\Phi}_{2}+\bar{\Phi}_{2}^{\mathrm{T}}, \quad \bar{\Phi}_{2}=\left[\begin{array}{lll}
L+S & -L+R & -R-S
\end{array}\right] .
$$

Furthermore, if there are no stochastic perturbations, the neural network (3.26) will reduce to

$$
d x(t)=\left[-A x(t)+W_{0} f(x(t))+W_{1} f(x(t-h(t)))\right] d t .
$$

High-order neural networks of the type (3.30) have been investigated intensively in the literature, for example in $[7,17,20,26]$. The following corollary provides a complementary method to the results in [7, 17, 26]. Furthermore, the following corollary is less restrictive than that in [20].

Corollary 3.8. Consider the dynamics of the neural network (3.30). The system is robustly, globally, exponentially stable if there exist scalars $\varepsilon_{1}>0$ and $\varepsilon_{2}>0$ and matrices $P>0, Q_{2}>0, Z_{3}>0$ and $Z_{4}>0$, and $(\mathrm{Y})$ such that the PWCLMIS which 
is constructed by (3.29) and the LMI

$$
\left[\begin{array}{ccccc}
\bar{\Omega}_{11} & P W_{0} & \Omega_{13} & P W_{1} & 0 \\
* & -\varepsilon_{1} I & 0 & 0 & 0 \\
* & * & -\varepsilon_{1} I & 0 & 0 \\
* & * & * & -\varepsilon_{2} I & 0 \\
* & * & * & * & \Omega_{77}
\end{array}\right]<0
$$

holds.

3.2. Exponential stability for uncertain systems The following theorem gives a sufficient condition of robustly exponentially stable in mean square for the network dynamics of (2.4).

THEOREM 3.9. Consider the dynamics of the high-order uncertain stochastic delayed neural network (2.4). The system is robustly, globally, exponentially stable in the mean square if there exist scalars $\rho>0, \varepsilon_{i}>0(i=1,2,3)$ and matrices $P>0, Q_{1}>0$, $Q_{2}>0, Z_{j}>0(j=1,2,3,4)$ and $(\mathrm{X})$ such that the PWCLMIS which is constructed by (3.2), (3.4) and the following LMI holds:

$$
\left[\begin{array}{cccccccccc}
\Omega_{11} & P W_{0} & \Omega_{13} & P W_{1} & P W_{2} & \rho \Sigma_{1}^{\mathrm{T}} & P M & -\varepsilon_{4} N_{1}^{\mathrm{T}} & 0 & 0 \\
* & -\varepsilon_{1} I & 0 & 0 & 0 & 0 & 0 & \varepsilon_{4} N_{2}^{\mathrm{T}} & 0 & 0 \\
* & * & -\varepsilon_{1} I & 0 & 0 & 0 & 0 & 0 & 0 & 0 \\
* & * & * & -\varepsilon_{2} I & 0 & 0 & 0 & \varepsilon_{4} N_{3}^{\mathrm{T}} & 0 & 0 \\
* & * & * & * & -\varepsilon_{3} I & 0 & 0 & 0 & 0 & 0 \\
* & * & * & * & * & -\rho I & 0 & 0 & 0 & 0 \\
* & * & * & * & * & * & -\varepsilon_{4} I & 0 & 0 & 0 \\
* & * & * & * & * & * & * & -\varepsilon_{4} I & 0 & 0 \\
* & * & * & * & * & * & * & * & \Omega_{77} & \rho \Sigma_{2}^{\mathrm{T}} \\
* & * & * & * & * & * & * & * & * & -\rho I
\end{array}\right]<0 .
$$

PROOF. From Theorem 3.6, the system (2.4) is robustly, globally, exponentially stable in mean square if there exist scalars $\rho>0$ and $\varepsilon_{i}>0(i=1,2,3)$ and matrices $P>0$, $Q_{1}>0, Q_{2}>0$ and $Z_{j}>0(j=1,2,3,4)$, and (X) such that the PWCLMIS which is constructed by (3.2), (3.4) and the LMI

$\left[\begin{array}{cccccccc}\tilde{\Omega}_{11} & P\left(W_{0}+\Delta W_{0}\right) & \Omega_{13} & P\left(W_{1}+\Delta W_{1}\right) & P W_{2} & \rho \Sigma_{1}^{\mathrm{T}} & 0 & 0 \\ * & -\varepsilon_{1} I & 0 & 0 & 0 & 0 & 0 & 0 \\ * & * & -\varepsilon_{1} I & 0 & 0 & 0 & 0 & 0 \\ * & * & * & -\varepsilon_{2} I & 0 & 0 & 0 & 0 \\ * & * & * & * & -\varepsilon_{3} I & 0 & 0 & 0 \\ * & * & * & * & * & -\rho I & 0 & 0 \\ * & * & * & * & * & * & \Omega_{77} & \rho \Sigma_{2}^{\mathrm{T}} \\ * & * & * & * & * & * & * & -\rho I\end{array}\right]<0$,

holds, where $\tilde{\Omega}_{11}=-(A+\Delta A) P-P(A+\Delta A)+Q_{1}+Q_{2}$. 
Defining

$$
\begin{aligned}
\hat{\Psi} & =\left[\begin{array}{cccccccc}
\Omega_{11} & P W_{0} & \Omega_{13} & P W_{1} & P W_{2} & \rho \Sigma_{1}^{\mathrm{T}} & 0 & 0 \\
* & -\varepsilon_{1} I & 0 & 0 & 0 & 0 & 0 & 0 \\
* & * & -\varepsilon_{1} I & 0 & 0 & 0 & 0 & 0 \\
* & * & * & -\varepsilon_{2} I & 0 & 0 & 0 & 0 \\
* & * & * & * & -\varepsilon_{3} I & 0 & 0 & 0 \\
* & * & * & * & * & -\rho I & 0 & 0 \\
* & * & * & * & * & * & \Omega_{77} & \rho \Sigma_{2}^{\mathrm{T}} \\
* & * & * & * & * & * & * & -\rho I
\end{array}\right], \\
c_{1}^{\mathrm{T}} & =\left[\begin{array}{llllllll}
M^{\mathrm{T}} P & 0 & 0 & 0 & 0 & 0 & 0 & 0
\end{array}\right] \text { and } \\
r_{1} & =\left[\begin{array}{llllllll}
-N_{1} & N_{2} & 0 & N_{3} & 0 & 0 & 0 & 0
\end{array}\right]
\end{aligned}
$$

and using (2.5) allows us to rewrite (3.33) as

$$
\hat{\Psi}+c_{1} F r_{1}+r_{1}^{\mathrm{T}} F^{\mathrm{T}} c_{1}^{\mathrm{T}}<0 .
$$

By Lemma 3.5, Equation (3.34) holds if and only if there is a scalar $\varepsilon_{4}>0$ such that

$$
\hat{\Psi}+\varepsilon_{4}^{-1} c_{1} c_{1}^{\mathrm{T}}+\varepsilon r_{1}^{\mathrm{T}} r_{1}<0
$$

It follows from the Schur complement lemma (Lemma 3.2) that (3.35) holds if and only if (3.39) holds. The proof of Theorem 3.9 is complete.

If only the discrete time delay appears in the neural network, (2.4) can be simplified to

$$
\begin{aligned}
d x(t)= & {\left[-(A+\Delta A) x(t)+\left(W_{0}+\Delta W_{0}\right) f(x(t))+\left(W_{1}+\Delta W_{1}\right) f(x(t-h(t)))\right] d t } \\
& +\sigma(t, x(t), x(t-h)) d w(t) .
\end{aligned}
$$

We have the following corollary.

Corollary 3.10. Consider the dynamics of the neural network (3.36). The system is robustly, globally, exponentially stable in mean square if there exist scalars $\rho>0$, $\varepsilon_{1}>0$ and $\varepsilon_{2}>0$ and matrices $P>0, Q_{2}>0, Z_{3}>0$ and $Z_{4}>0$, and $(\mathrm{Y})$ such that the PWCLMIS which is constructed by (3.27), (3.29) and the LMI

$$
\left[\begin{array}{ccccccccc}
\Omega_{11} & P W_{0} & \Omega_{13} & P W_{1} & \rho \Sigma_{1}^{\mathrm{T}} & P M & -\varepsilon_{4} N_{1}^{\mathrm{T}} & 0 & 0 \\
* & -\varepsilon_{1} I & 0 & 0 & 0 & 0 & \varepsilon_{4} N_{2}^{\mathrm{T}} & 0 & 0 \\
* & * & -\varepsilon_{1} I & 0 & 0 & 0 & 0 & 0 & 0 \\
* & * & * & -\varepsilon_{2} I & 0 & 0 & \varepsilon_{4} N_{3}^{\mathrm{T}} & 0 & 0 \\
* & * & * & * & -\rho I & 0 & 0 & 0 & 0 \\
* & * & * & * & * & -\varepsilon_{4} I & 0 & 0 & 0 \\
* & * & * & * & * & * & -\varepsilon_{4} I & 0 & 0 \\
* & * & * & * & * & * & * & \Omega_{77} & \rho \Sigma_{2}^{\mathrm{T}} \\
* & * & * & * & * & * & * & * & -\rho I
\end{array}\right]<0
$$

holds. 
If there are no stochastic perturbations $\sigma(t, x(t), x(t-h))$, the neural network (3.36) will be reduced to

$$
\begin{aligned}
d x(t)= & {\left[-(A+\Delta A) x(t)+\left(W_{0}+\Delta W_{0}\right) f(x(t))\right.} \\
& \left.+\left(W_{1}+\Delta W_{1}\right) f(x(t-h(t)))\right] d t .
\end{aligned}
$$

We have the following corollary.

COROLlary 3.11. Consider the dynamics of the neural network (3.38). The system is robustly, globally, exponentially stable if there exist scalars $\varepsilon_{1}>0$ and $\varepsilon_{2}>0$ and matrices $P>0, Q_{2}>0, Z_{3}>0$ and $Z_{4}>0$, and $(\mathrm{Y})$ such that the PWCLMIS which is constructed by (3.29) and the LMI

$$
\left[\begin{array}{ccccccc}
\Omega_{11} & P W_{0} & \Omega_{13} & P W_{1} & P M & -\varepsilon_{4} N_{1}^{\mathrm{T}} & 0 \\
* & -\varepsilon_{1} I & 0 & 0 & 0 & \varepsilon_{4} N_{2}^{\mathrm{T}} & 0 \\
* & * & -\varepsilon_{1} I & 0 & 0 & 0 & 0 \\
* & * & * & -\varepsilon_{2} I & 0 & \varepsilon_{4} N_{3}^{\mathrm{T}} & 0 \\
* & * & * & * & -\varepsilon_{4} I & 0 & 0 \\
* & * & * & * & * & -\varepsilon_{4} I & 0 \\
* & * & * & * & * & * & \Omega_{77}
\end{array}\right]<0
$$

holds.

\section{Numerical examples}

EXAMPle 1. Consider a two-neuron stochastic neural network (2.1) with both discrete and distributed delays, where $d_{\tau}=0.5, d_{h}=0.5, L=1.2$ and

$$
\begin{array}{cc}
A=\left[\begin{array}{cc}
1.2 & 0 \\
0 & 1.2
\end{array}\right], \quad W_{0}=\left[\begin{array}{cc}
1.5 & -1.6 \\
-1.6 & 1.5
\end{array}\right], \quad W_{1}=\left[\begin{array}{ll}
1.2 & 0.3 \\
0.3 & 0.9
\end{array}\right], \\
W_{2}=\left[\begin{array}{cc}
0.16 & -0.64 \\
-0.64 & 0.16
\end{array}\right], \\
\Sigma_{\mu}=\left[\begin{array}{cc}
0.2 & 0 \\
0 & 0.2
\end{array}\right], \quad \Sigma_{1}=\left[\begin{array}{cc}
0.08 & 0 \\
0 & 0.08
\end{array}\right] \text { and } \quad \Sigma_{2}=\left[\begin{array}{cc}
0.09 & 0 \\
0 & 0.09
\end{array}\right] .
\end{array}
$$

Table 1 gives the maximum allowable value $h$ for different $\tau$ determined by Theorem 3.6.

TABLE 1. Maximum $h$ calculated for various $\tau$ from Theorem 3.6 for Example 1 .

\begin{tabular}{lc}
\hline$\tau$ & Maximum allowable value of $h$ \\
\hline $1.0000 \times 10^{20}$ & $3.1467 \times 10^{15}$ \\
$1.0000 \times 10^{15}$ & $1.3350 \times 10^{21}$ \\
$1.0000 \times 10^{10}$ & $5.0000 \times 10^{20}$ \\
\hline
\end{tabular}


However, by applying Theorem 1 of [20], we gain the maximum allowable value $\tau=1.7855$. At the same time, Theorem 1 of [20] is discrete time-delay $h$ independent.

EXAMPLE 2. Consider a two-neuron uncertain stochastic neural network (2.4) with mixed delays, where $d_{\tau}=0.5, d_{h}=0.5, L=1.2$ and

$$
\begin{gathered}
A=\left[\begin{array}{cc}
0.4 & 0 \\
0 & 0.4
\end{array}\right], \quad W_{0}=\left[\begin{array}{cc}
0.1 & -0.2 \\
-0.2 & 0.1
\end{array}\right], \quad W_{1}=\left[\begin{array}{ll}
0.2 & 0.3 \\
0.3 & 0.1
\end{array}\right], \\
W_{2}=\left[\begin{array}{cc}
0.1 & -0.64 \\
-0.64 & 0.1
\end{array}\right], \\
\Sigma_{\mu}=\left[\begin{array}{cc}
0.1 & 0 \\
0 & 0.1
\end{array}\right], \quad \Sigma_{1}=\left[\begin{array}{cc}
0.01 & 0 \\
0 & 0.01
\end{array}\right], \quad \Sigma_{2}=\left[\begin{array}{cc}
0.04 & 0 \\
0 & 0.04
\end{array}\right], \\
M=N_{2}=\left[\begin{array}{cc}
0.1 & 0 \\
0 & 0.2
\end{array}\right] \quad \text { and } \quad N_{1}=N_{3}=\left[\begin{array}{cc}
0.2 & 0 \\
0 & 0.1
\end{array}\right] .
\end{gathered}
$$

Table 2 gives the maximum allowable value $h$ for different $\tau$ determined by Theorem 3.9.

TABLE 2. Maximum $h$ calculated for various $\tau$ from Theorem 3.9 for Example 2.

\begin{tabular}{lc}
\hline$\tau$ & Maximum allowable value of $h$ \\
\hline $1.0000 \times 10^{20}$ & $5.6448 \times 10^{14}$ \\
$1.0000 \times 10^{15}$ & $1.2302 \times 10^{20}$ \\
$1.0000 \times 10^{10}$ & $2.8015 \times 10^{19}$ \\
\hline
\end{tabular}

From Theorems 3.6 and 3.9, the admissible mixed time delays are large. We are able to obtain such large mixed time delays because of the PWCLMIS approach and the fact that the value fields of time delays in the PWCLMIS are free. In the PWCLMIS, all system parameters $A, W_{0}, W_{1}, W_{2}, \Sigma_{\mu}, \Sigma_{1}, \Sigma_{2}, M, N_{1}, N_{2}$ and $N_{3}$ are in one LMI and the time delays $\tau$ and $h$ in the other which is without system parameters. At the same time, there are free-weighting matrices $H, J, K, L, R, S$ in the latter LMI. So the value fields of the time delays are large (or free).

\section{Conclusion}

This paper has proposed new sufficient conditions of global exponential stability for deterministic and uncertain stochastic high-order neural networks. These conditions are discrete and distributed time-varying delay-dependent conditions. The concept of a PWCLMIS has been developed. The criteria have been developed in terms of the PWCLMIS, by which large mixed time delays can be achieved. The criteria are more general than those in some recent works. Two numerical examples have been given to demonstrate the merit of the criteria presented. 


\section{Acknowledgements}

This work is partially supported by the National 863 Key Program of China (2008AA042902), the Doctor Base Foundation of Colleges and Universities by the Ministry of Education of China (200802550007), the Key Scientific Research and Innovation Program of Shanghai Education Committee (09zz66) and the Key Basic Research Program of Shanghai City (09JC1400700).

\section{References}

[1] S. Arik, "Global robust stability analysis of neural networks with discrete time delays", Chaos Solitons Fractals 26 (2005) 1407-1414.

[2] E. Artyomov and O. Yadid-Pecht, "Modified high-order neural network for invariant pattern recognition", Pattern Recognit. Lett. 26 (2005) 843-851.

[3] S. Blythe, X. Mao and X. Liao, "Stability of stochastic delay neural networks", J. Franklin Inst. 338 (2001) 481-495.

[4] S. Boyd, L. El Ghaoui, E. Feron and V. Balakrishnan, Linear matrix inequalities in system and control theory (SIAM, Philadelphia, PA, 1994).

[5] J. Cao and T. Chen, "Globally exponentially robust stability and periodicity of delayed neural networks", Chaos Solitons Fractals 22 (2004) 957-963.

[6] J. Cao, D.-S. Huang and Y. Qu, "Global robust stability of delayed recurrent neural networks", Chaos Solitons Fractals 23 (2005) 221-229.

[7] J. Cao, J. Liang and J. Lam, "Exponential stability of high-order bidirectional associative memory neural networks with time delays", Phys. D 199 (2004) 425-436.

[8] A. Dembo, O. Farotimi and T. Kailath, "High-order absolutely stable neural networks", IEEE Trans. Circuits. Syst. 38 (1991) 57-65.

[9] A. Friedman, Stochastic differential equations and their applications, Volume 2 (Academic Press, New York, 1976).

[10] K. Gu, "An integral inequality in the stability problem of time-delay systems", Proc. 39th IEEE Conf. on Decision and Control, Sydney, Australia, December 2000 (IEEE Computer Society, Los Alamitos, CA) 2805-2810.

[11] J. K. Hale, Theory of functional differential equations (Springer, New York, 1977).

[12] H. Huang, D. W. C. Ho and J. Lam, "Stochastic stability analysis of fuzzy Hopfield neural networks with time-varying delays", IEEE Trans. Circuits Syst.: Part II 52 (2005) 251-255.

[13] N. B. Karayiannis and A. N. Venetsanopoulos, "On the training and performance of high-order neural networks", Math. Biosci. 129 (1995) 143-168.

[14] M. Li, W. Zhou, H. Wang, Y. Chen, R. Lu and H. Lu, "Delay-dependent robust $H_{\infty}$ control for uncertain stochastic systems", Proc. 17th IFAC World Congress, Seoul, Korea, July 2008 (Curran Associates, New York, 2009) 6004-6009.

[15] I. R. Petersen, "A stabilization algorithm for a class of uncertain linear systems", Systems Control Lett. 8 (1987) 351-357.

[16] D. Psaltis, C. H. Park and J. Hong, "Higher order associative memories and their optical implementations", Neural Networks 1 (1988) 143-163.

[17] F. Ren and J. Cao, "LMI-based criteria for stability of high-order neural networks with timevarying delay", Nonlinear Anal. Ser. B: Real World Appl. 7 (2006) 967-979.

[18] S. Ruan and R. S. Filfil, "Dynamics of a two-neuron system with discrete and distributed delays", Phys. D 191 (2004) 323-342.

[19] L. Wan and J. Sun, "Mean square exponential stability of stochastic delayed Hopfield neural networks", Phys. Lett. A 343 (2005) 306-318.

[20] Z. Wang, J. Fang and X. Liu, "Global stability of stochastic high-order neural networks with discrete and distributed delays", Chaos Solitons Fractals 36 (2008) 388-396. 
[21] Z. Wang, S. Lauria, J. Fang and X. Liu, "Exponential stability of uncertain stochastic neural networks with mixed time-delays", Chaos Solitons Fractals 32 (2007) 62-72.

[22] Z. Wang, Y. Liu and X. Liu, "On global asymptotic stability of neural networks with discrete and distributed delays", Phys. Lett. A 345 (2005) 299-308.

[23] Z. Wang, H. Shu, Y. Liu, D. W. C. Ho and X. Liu, "Robust stability analysis of generalized neural networks with discrete and distributed time delays", Chaos Solitons Fractals 30 (2006) 886-896.

[24] Z. Wu, H. Su, J. Chu and W. Zhou, "New results on robust exponential stability for discrete recurrent neural networks with time-varying delays", Neurocomputing 72 (2009) 3337-3342.

[25] Z. Wu, H. Su, J. Chu and W. Zhou, "Improved result on stability analysis of discrete stochastic neural networks with time delay", Phys. Lett. A 373 (2009) 1546-1552.

[26] L. Xie, "Output feedback $H_{\infty}$ control of systems with parameter uncertainty", Internat. $J$. Control. 63 (1996) 741-750.

[27] H. Zhao, "Existence and global attractivity of almost periodic solution for cellular neural network with distributed delays", Appl. Math. Comput. 154 (2004) 683-695.

[28] W. Zhou, H. Lu and C. Duan, "Exponential stability of hybrid stochastic neural networks with mixed time delays and nonlinearity", Neurocomputing 72 (2009) 3357-3365. 